\title{
Relation between BCG vaccine scar and an interferon-gamma release assay in immigrant children with "positive" tuberculin skin test $(\geq 10 \mathrm{~mm})$
}

Margret Johansson Gudjónsdóttir ${ }^{1,4^{*}}$, Karsten Kötz ${ }^{1}$, Ruth Stangebye Nielsen ${ }^{1}$, Philip Wilmar ${ }^{1}$, Sofia Olausson², Daniel Wallmyr ${ }^{3}$ and Birger Trollfors ${ }^{1}$

\begin{abstract}
Background: Immigrants from countries with high incidence of tuberculosis (TB) are usually offered screening when they arrive to low incidence countries. The tuberculin skin test (TST) is often used. The interferon gamma release assays (IGRAs) are more specific and not affected by BCG vaccination. The aims of this study were 1. To see if there if there is a correlation between a positive IGRA (QFT) and presence of a BCG scar in children with TST $\geq 10 \mathrm{~mm}, 2$. To compare the TST diameter with QFT result, 3. To see if chest X-ray can be omitted in QFT negative children despite TST $\geq 10 \mathrm{~mm}$.

Methods: 762 healthy children/adolescents (median age 14 years) arriving to Gothenburg and surroundings with TST $\geq 10 \mathrm{~mm}$ were tested with QFT.

Results: A total of 163/492 (33 \%) children with BCG scar had positive QFT, whereas 205/270 (76 \%) without BCG scar had positive QFT $(p<0.0001)$. The median TST was $12 \mathrm{~mm}$ in QFT negative and $18 \mathrm{~mm}$ in QFT positive children $(p<0.0001)$ but with considerable overlap. Median TST was the same $(12 \mathrm{~mm})$ in QFT negative children with and without BCG scar. Among the QFT positive children 25/368 had chest X-ray changes compared to 2/393 among the QFT negative children $(p<0.0007)$.

Conclusions: Previous BCG vaccination had an effect on the TST diameter so an IGRA is recommended to diagnose latent TB. Using only TST for screening of latent TB would lead to overdiagnosis. The TST diameter was larger in QFT positive than in QFT negative children but could not predict QFT in the individual patient. Chest $X$ ray contributes little to the diagnosis of TB in QFT negative children but can not be omitted because of late seroconversion of QFT in some patients.
\end{abstract}

Trial registration: Not applicable.

Keywords: Tuberculosis, BCG scar, Tuberculin skin test, Quantiferon

\footnotetext{
* Correspondence: margret.johansson.gudjonsdottir@vgregion.se

${ }^{1}$ Department of Paediatrics, Institute of Clinical Sciences, Sahlgrenska

Academy, Gothenburg University, Gothenburg, Sweden

${ }^{4}$ Department of Pediatrics, Neonatology, Sahlgrenska University Hospital,

41685 Gothenburg, Sweden

Full list of author information is available at the end of the article
} 


\section{Background}

Immigrants from countries with high incidence of tuberculosis (TB) are offered medical examination and screening for $\mathrm{TB}$ and other infectious diseases when they arrive to Sweden. The screening for TB consisted until recently of physical examination and a tuberculin skin test (TST). If TST was $\geq 10 \mathrm{~mm}$ a chest $\mathrm{X}$ ray was performed. If there were no signs of active TB, preventive chemotherapy was recommended to children and adolescents (in the following called children) if compliance could be expected to be good. From 2007 children coming to the Gothenburg area with TST $\geq 10 \mathrm{~mm}$, irrespective if a BCG scar was visible or not, were referred to clinics specialising in TB where Quantiferon was assayed. Preventive chemotherapy was offered if Quantiferon was positive. The TST limit of $10 \mathrm{~mm}$ follows the recommendations of the National Board of Health and Welfare.

TST is not ideal for screening for latent TB. It requires two visits and it crossreacts with antigens in the Bacillus Calmette-Guérin (BCG) vaccine and with many nontuberculous mycobacteria (NTM) [1-8]. Repeated testing can induce false positive results. Furthermore, the definition of a positive test varies from 5 to $15 \mathrm{~mm}$ ) [1-8]. The US Centers for Disease Control and Prevention adjust for underlying diseases, history of vaccination and origin of the immigrant (http://www.cdc.gov/tb/publications/factsheets/testing/ skintesting.htm). In the UK, $5 \mathrm{~mm}$ is used as cut-off in children with BCG scar (National Institure for Health and Care Excellence, https://www.nice.org.uk/ guidance/ng33/chapter/recommen dations\# diagnosinglatent-tb-in-children-and-young-people).

The interferon gamma release assays (IGRAs) solve many, but not all, problems in the evaluation of latent TB. They require only one visit, do not cross-react with BCG vaccines and cross-react with fewer NTM than TST. Several studies have shown a rather good correlation between TST and IGRAs except in very young children [9-13] but a recent study in 126 adults showed a relatively poor correlation between TST and IGRAs and also between two IGRAs (QuantiFERON -TB Gold In-Tube ${ }^{\bullet}(\mathrm{QFT})$ and T-SPOT.TB $\left.{ }^{\circ}\right)$ [12]. The use of IGRAs is therefore controversial and has not replaced TST for diagnosis of latent TB in many countries $[14,15]$.

It is usually difficult to obtain a reliable vaccination history from the accompanying adult family member and impossible if the child arrives alone to Sweden. The only way to obtain information about BCG vaccination is to look for typical BCG scars even though BCG vaccination does not always result in a scar and scars can wane with time [1, 16-18].

\section{Methods}

\section{The aim of the study}

The three questions in the present study were; 1 . Is there a correlation between presence of a BCG scar and positive QFT? (The presence of a BCG scar was in the absence of documented BCG vaccination used as a substitute for vaccination), 2. Does the size of the TST differ between children with positive and negative QFT? 3 . Can chest $\mathrm{X}$ ray be omitted in children with negative QFT, as has been suggested for adults [12]?

\section{Study design and setting}

The study is a retrospective evaluation of patient records of immigrant children who had undergone health examination and been found to have TST $\geq 10 \mathrm{~mm}$. The initial health examination including TST of immigrant children in the area is performed at several Primary Health Clinics. Children with TST $\geq 10 \mathrm{~mm}$ are referred to the Departments of Pediatrics of Sahlgrenska University Hospital or the outpatient clinics in Angered (north-east Gothenburg), Borås or Alingsås (all serving surrounding communities). A chest X-ray is performed. The study included children examined from December 2007 through May 2016. The inclusion criteria in the study were 1) TST $\geq 10 \mathrm{~mm}, 2$ ) No clinical symptoms (cough, swollen lymph nodes, loss of weight and appetite, fatigue, fever) or findings on physical examination compatible with active tuberculosis, 3) No known exposure to tuberculosis before or after arrival to Sweden. Three children, who had come to Sweden alone, who told that they had been treated for many months in their home countries with medicines which made their urine red (assumed to be Rifampicin), were not included. One child was excluded because it had several scars after recent varicella. It was not possible to evaluate if any of these scars were caused by BCG. There are no reliable statistics about the number of immigrant children who undergo health examinations in the area but an estimate indicates that it is about 600 children (70\% of all immigrant children) per year increasing the last years (personal communication: Leif Dotevall, MD, Department of Communicable Diseases, Västra Götaland, Sweden).

\section{Patients}

A total of 762 children were included in the study. Gender and age distribution is shown in Table 1. As many as 215 children came alone without an accompanying adult (Table 1). Most of the unaccompanied minors came from Somalia (121), Afghanistan (48) and Eritrea (16). These children were all aged between 11 and 18 years.

Chest $\mathrm{X}$ ray was performed in all but one child, whose mother declined X ray. This child had a scar and was QFT negative. 
Table 1 Base-line characteristics of the 762 children included in the study

\begin{tabular}{ll}
\hline Boys-girls & 458 boys, 304 girls \\
Median age & 14 years \\
10-18 years & 625 \\
5-9 years & 99 \\
0-4 years & 38 \\
Median time ${ }^{\text {a }}$ spent in Sweden & 8 (0-96) months \\
Unaccompanied minors & 215 (46 girls, 169 boys) \\
Concomitant diseases & 36 \\
HBsAg positive & 8 \\
Asthma & 3 \\
Rickets & 3 \\
Kidney stones & 4 \\
Epilepsia & 3 \\
Iron deficiency anema & 3 \\
Thalassemia minor & 2 \\
Mb Down & 2 \\
Coeliaci & 1 \\
Psychomotor retardation & 1 \\
Duchenne's muscular dystrophy & 1 \\
Diabetes mellitus & 1 \\
Cannabis smoker & 1 \\
Mb Crohn & 1 \\
Cerebral palsy & 1 \\
& \\
aime (range) when QFT was assayed & \\
\hline
\end{tabular}

Both arms were inspected for scars compatible with BCG vaccination. If no scars were found on the arms, the thighs were inspected except in a few teen-age girls who refused this. They all said that they had no scars on the legs, which was accepted as absence of scar. The evaluation of BCG scar was made before the QFT result was known. Only four children had written documentation of BCG vaccination in their home countries. One of them had no scar and is included among the children without scar. The other three had a scar. Three children had scars on the dorsal side of the lower arm and one on the hip. They were all accompanied by their mothers who knew that these scars were induced by vaccination against TB.

The median time in Sweden was 8 (range 0-96) months when the QFT was performed. All patients were tested for syphilis, hepatitis B and HIV. No patient was syphilis or HIV infected. There were 36 children with chronic diseases (Table 1). The eight HBsAg positive patients were all asymptomatic and had normal liver function tests.

The children came from 59 different countries, when political borders and not ethnicity was considered.
Chechnya and Dagestan was included in Russia and Kosovo in Serbia. Kurds and Romanis were included in the countries they came from. The only exception was 23 children from the Ogaden region, Ethiopia, who were included among Somalians. The largest groups came from Somalia + Ogaden (349), Afghanistan (77), Iraq (34) and Eritrea (24). Table 2 shows numbers of children from different regions of the world and the presence of BCG scars.

\section{Tuberculin skin test}

$0.1 \mathrm{ml}$ containing 2 TU PPD RT 23 (Statens Seruminstitut, Copenhagen, Denmark) was injected intracutaneously into the dorsal side of the lower arm. The diameter of the swelling was measured after $72 \mathrm{~h}$. Towards the end of the study there was a lack of the TST reagents from Statens Seruminstitut. During that time Tubertest from Sanofi, Lyon, France was used.

\section{Quantiferon}

Blood for QFT was obtained $\geq 2$ months after TST was read. The sampling and analyses were performed with QuantiFERON ${ }^{\circ}$-TB Gold In Tube according to the instructions of the manufacturer (Cellestis Ltd., Chadstone, Victoria, Australia). One $\mathrm{mL}$ blood was collected into each of three different collection tubes, which include Nil Control tube, TB Antigen tube, and Mitogen (positive control) tube. The samples were transported to the laboratory within $16 \mathrm{~h}$. At the laboratory, the collection tubes were incubated at $37^{\circ}$ for $16-20 \mathrm{~h}$ and subsequently centrifuged at $2300 \mathrm{~g}$ for $15 \mathrm{~min}$. The concentration of interferon gamma in the resulting plasma in the tubes was measured by ELISA and related to a concentration standard. The absorbance was measured in Vmax Kinetic Microplate Reader (Molecular Devices, USA) and the optical density (OD) values were evaluated using the $\mathrm{QFT}^{\oplus}-\mathrm{TB}$ Gold Analysis Software version 2.50. According to the producer, QFT is positive when TB-antigen - Nil is $\geq 0.35 \mathrm{U} / \mathrm{mL}$. In this study new blood samples were obtained and the test was repeated when TB-antigen - Nil was 0.35 to $\leq$

Table 2 Frequency of BCG scars

\begin{tabular}{lll}
\hline & $\begin{array}{l}\text { Number with scar/total } \\
\text { number from this region }\end{array}$ & $\begin{array}{l}\text { Proportion } \\
\text { with scar }\end{array}$ \\
\hline Somalia & $193 / 349$ & $55 \%$ \\
Middle East + North Africa & $140 / 190$ & $74 \%$ \\
Rest of Africa & $57 / 99$ & $58 \%$ \\
South East Asia & $40 / 49$ & $82 \%$ \\
Europe & $41 / 50$ & $82 \%$ \\
China + Taiwan + Mongolia & $9 / 11$ & $82 \%$ \\
India + Nepal & $5 / 7$ & $71 \%$ \\
South America & $7 / 7$ & $100 \%$ \\
\hline
\end{tabular}

Numbers and proportions of children with BCG scar from different parts of the world 
$1.0 \mathrm{U} / \mathrm{mL}$. This was seen in 18 patients. Seventeen of them were clearly negative in the second test and one was clearly positive. The results of the second test were used in the study.

\section{Statistics}

Proportions were compared with 2-tailed Fisher's exact test using the method of summing small $\mathrm{P}$ values and median values with 2-tailed Mann-Whitney $U$ test.

\section{Results}

A total of 762 children fulfilled all inclusion criteria. BCG scars were found in 492 of them (65\%) (Table 2). Scars were found in significantly lower proportions in children from Somalia and the rest of Sub-Saharan Africa (56 \%) than in all other children combined $(77 \%)(p<0.0001)$.

QFT was positive in 163/492 (33\%) children with a BCG scar and in 205/270 (76 \%) without a scar ( $p<$ $0.0001)$. Significant differences were found in children from Somalia, the Middle East + North Africa, Sub-Saharan Africa, Southeast Asia, Europe and China. Children from India and South America were too few for separate calculations (Table 3). Somalian children with a BCG scar were significantly more often QFT positive (55\%) than all other groups combined $(22 \%)(p<0.0001)$. Significant differences of the same magnitude were found in boys and girls.

Among children with a scar and TST $\geq 10 \mathrm{~mm} \mathrm{329/}$ 492 (67 \% had negative QFT, while only 65/270 (24\%) of the children without a scar and TST $\geq 10 \mathrm{~mm}$ had negative QFT $(p<0.0001)$.

Among children 5 - 9 years 19/58 (33\%) with BCG scar had positive QFT compared to 28/41 (68 \%) children without scar $(p=0.0006)$. A statistical comparison for children $<5$ years of age was not meaningful because
34/36 with scar had negative QFT while one of two without scar had negative QFT.

Among the 215 unaccompanied children aged 11 18 years 131/215 (61 \%) had positive Quantiferon compared to $179 / 383$ (47 \%) children aged $11-18$ years who came with their families $(p=0.0009)$. Among those who came with their families and were aged between 11 and 18 years, the proportion with BCG scars was higher 266/ $383(69 \%)$ than in the unaccompanied children, 113/215 $(53 \%)(p<0.0001)$.

The median TST diameters were significantly higher in QFT positive than in QFT negative children $(p<0.0001)$ both in children with and without scar (Table 4). There was, however, a considerable overlap (Fig. 1). Among the children with negative QFT, 279 had TST 10-14 mm and 115 had TST $\geq 15 \mathrm{~mm}$.

Chest $X$ ray changes were seen in 25/368 QFT positive and in 2/394 QFT negative children. The changes consisted of enlarged hilar or tracheal lymph nodes or mild to moderate inflammatory infiltrates. One QFT negative child (6 year old boy from Somalia) had a TST just at the cut-off value of $10 \mathrm{~mm}$. No mycobacteria could be isolated from bronchoalveolar lavage. He had elevated erythrocyte sedimentation rate (ESR) and C- Reactive Protein Test (CRP). His changes disappeared after 6 months of antituberculosis treatment for active TB with initially four drugs. This QFT negative child became QFT positive after 1.5 years, when he had finished treatment for active TB. The other QFT negative child (15 years old boy from Somalia) had a small basal inflammatory infiltrate. He had both ESR $<15 \mathrm{~mm} / \mathrm{h}$ and $\mathrm{CRP}<1.0 \mathrm{mg} / \mathrm{L}$ on repeated testing. He became QFT positive after 7 weeks. No mycobacteria were isolated from bronchoalveolar lavage. He was also treated for active TB with 4 drugs initially and chest $\mathrm{X}$ ray normalised.

Table 3 Relation between BCG scar and outcome of Quantiferon

\begin{tabular}{llll}
\hline & With scar & Without scar & \\
& Pos QFT/total number & Pos QFT/total number & $<$-value \\
\hline Somalia & $97 / 193(50 \%)$ & $129 / 156(83 \%)$ & $<0.0001$ \\
Middle East + North Africa & $34 / 140(24 \%)$ & $34 / 50(68 \%)$ & $<0.0001$ \\
Rest of Africa & $17 / 57(30 \%)$ & $27 / 42(64 \%)$ & 0.001 \\
South East Asia & $10 / 40(25 \%)$ & $8 / 9(89 \%)$ & 0.0007 \\
Europe & $4 / 41(10 \%)$ & $4 / 9(44 \%)$ & 0.0264 \\
China + Taiwan + Mongolia & $0 / 9(0 \%)$ & $2 / 2(100 \%)$ & 0.0182 \\
India + Nepal & $0 / 5(0 \%)$ & $1 / 2(50 \%)$ & $\mathrm{ns}$ \\
South America & $1 / 7(14 \%)$ & 0 & $\mathrm{~ns}$ \\
All boys & $90 / 291(31 \%)$ & $127 / 167(76 \%)$ & $<0.0001$ \\
All girls & $73 / 201(36 \%)$ & $78 / 103(76 \%)$ & $<0.0001$ \\
ALL CHILDREN & $163 / 492(33 \%)$ & $205 / 270(76 \%)$ & $<0.0001$ \\
\hline Postive & \\
\hline
\end{tabular}

Positive Quantiferon (number out of total (\%) with positive QFT in children with and without scar arriving to Sweden from different parts of the world. All children had TST $\geq 10 \mathrm{~mm}$. (p-values refer to comparisons of proportions in children with and without BCG scar in the respective population) 
Table 4 TST diameter related to BCG scar and Quantiferon

\begin{tabular}{llll}
\hline & QFT Negative & QFT Positive & $p$-value \\
\hline With BCG scar & $12(10-27)$ & $17(10-38)$ & $<0.0001$ \\
Without BCG scar & $12(10-23)$ & $18(10-40)$ & $<0.0001$ \\
\hline
\end{tabular}

Relation between median (range) TST diameter $(\mathrm{mm})$ and QFT result in children with and without BCG scar and positive and negative Quantiferon

Bronchoalveolar lavage was performed in 22 of the 25 QFT positive children with X ray changes. Mycobacterium tuberculosis was cultured from one of them. He was treated as a case of active TB and chest X-ray normalised. The 21 culture-negative children all had normal ESR and CRP. All of them were treated as active TB and all had normal chest $\mathrm{X}$ ray after 6 months.

\section{Discussion}

The most important difficulty in comparing TST and QFT is due to the absence of a "golden standard" for the diagnosis of latent TB. Based on theoretical assumptions a positive QFT is, however, more specific than a large TST which has no generally approved definition of "positive" and no positive and negative controls. This must be kept in mind when the present and a very large number of comparative studies are evaluated.

The study showed that there is a highly significant correlation between presence of BCG scar and positive
QFT. The reason for this could not be answered by the results. The finding that a higher proportion of children with scar had negative QFT than children without scar (67\% verus $24 \%$ ) speaks in favour of the possibility that BCG vaccination resulting in a scar influences TST while the finding of the same TST diameter in QFT negative children with and without scar speaks against an effect of BCG scar on TST (12 mm in both groups).

Assuming that a negative QFT makes latent TB unlikely something else may have induced the large TST of $\geq 10 \mathrm{~mm}$ in many QFT negative children. This could be infections with NTM, which is unlikely because NTM would hardly give any difference between children with and without scar. Another possibility is previous TST testing with booster effect (of which we could get no information), which is also unlikely because in lowincome countries with ongoing civil wars, latent TB is hardly looked for. The difference between children with and without a BCG scar makes BCG vaccination resulting in a scar the most likely possibility, even though the causes might be multifactorial. This result was unrelated to country of origin, gender and to age $\geq 10$ years and 5-9 years. The great majority of children with a BCG scar can be assumed to have been vaccinated at birth or soon afterwards, since the World Health Organisation recommends BCG vaccination as early as possible. Since

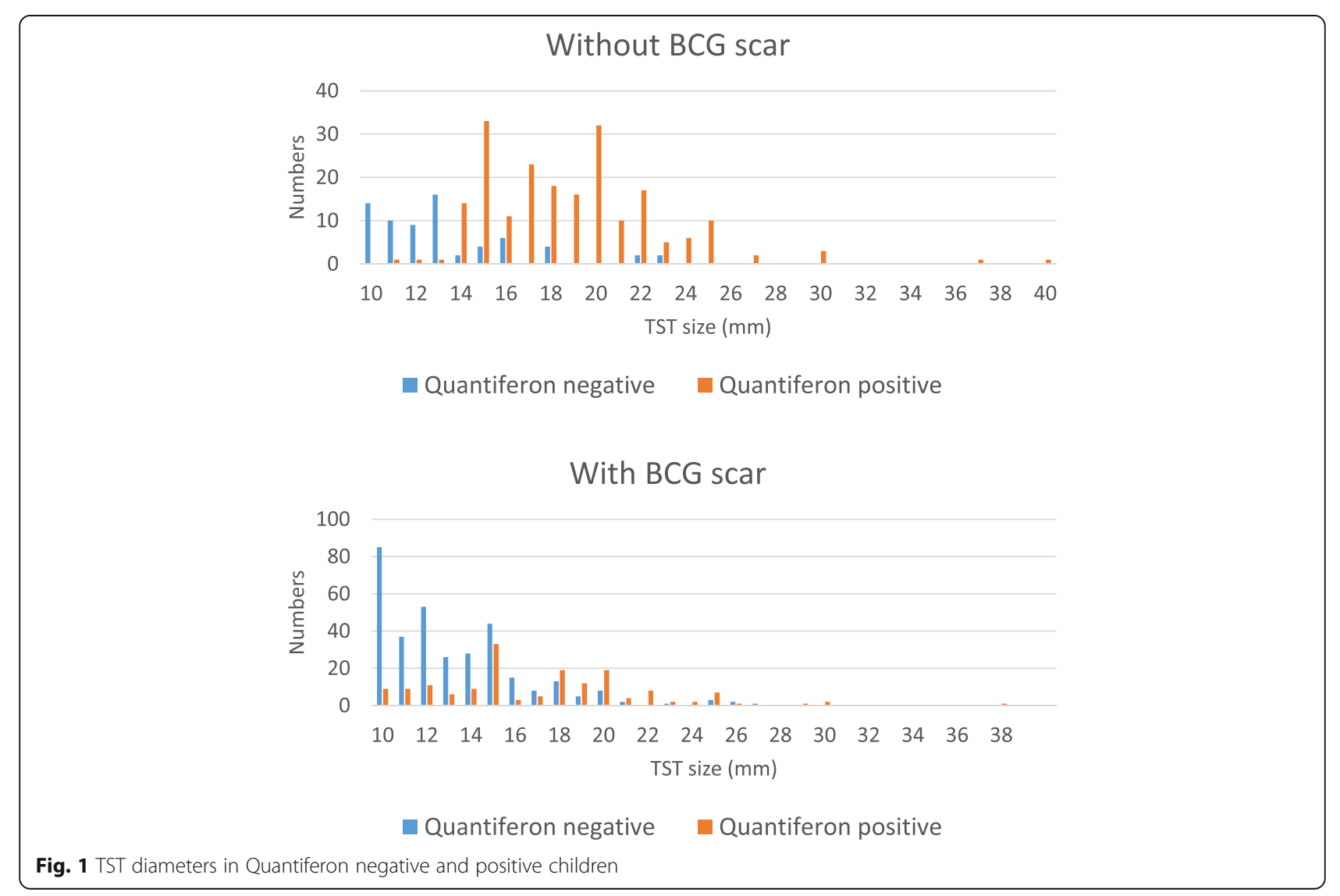


most of the children in the present study were adolescents the effect of BCG on TST seems to last for several years. This finding is in contrast with a study of school children in India stating that TST can be used for survey of TB irrespective of BCG vaccination [19] and a study from Spain indicating that BCG can give a false positive TST result in children $<3$ years but not in older children [20]. The most likely explanation of our finding is that BCG vaccination can induce an increase in the TST reaction, while QFT is unaffected by BCG vaccination $[1-8,20,21]$.

The reasons why some children with no scar have TST $\geq 10 \mathrm{~mm}$ and negative QFT could be that they had received a vaccination without getting a scar $[1,16-18]$ or that the scar had waned with time which according to a study from India may occur in $30 \%$ after a five year period [22]. Besides, different BCG vaccines have different ability to induce a scar [23, 24]. Their TST might still react with antigens in BCG. Another possibility could be that the children had been infected with NTM, many of which can induce an increase in TST without cross-reacting with the antigens in QFT [2-8]. Besides in patients with active TB negative QFT is found in about $20 \%[25,26]$. In the absence of a reference diagnostic method for latent TB, it is not possible to state how common falsely negative QFT is in patients with latent TB.

The median TST diameter was significantly larger in QFT positive than in QFT negative children but the overlap was great, so it is not possible to predict the result of QFT based on the size of the TST diameter in the individual case, which agrees with two previous studies of Turkish adults and of immigrant children in Norway $[27,28]$.

A British study of adults, most of them from Asia, showed that chest X-ray at the port of entry contributed little or nothing to finding TB cases [12]. The present study in children agreed only partly with this. Although chest $\mathrm{X}$ ray findings were significantly more common in QFT positive than in QFT negative children, chest $\mathrm{X}$ ray in only QFT positive children would have led to missed or delayed diagnosis in two children, who had late QFT conversions. Besides there were very few children $<5$ years. In this age group immunologic tests like IGRA may not work well.

The study shows that TST with a cut-off value between positive and negative of $10 \mathrm{~mm}$ should not be used alone for the diagnosis of latent TB. This would have led to an overdiagnosis of more than two-fold in children with a BCG scar and of one-third in children without a BCG scar, in the present study. Considering the costs of prophylactic medication, side effects and controls of adherence this seems unacceptable. Confirmation with the more specific IGRA's seems necessary both in vaccinated and nonvaccinated children. A Canadian study performed among North American Indians also concluded that TST alone is unsatisfactory for diagnosing latent TB in school children [8]. A two-step procedure with screening using the less expensive TST followed by an IGRA may be justifiable if compliance of return for reading the TST is high. A study from the United Kingdom advocates IGRA without preceding TST [12]. The U.K. study differs in two respects from the present. The immigrants in the U.K. study were mainly young adults coming from the Indian subcontinent and other Asian countries without civil wars while the patients in the present study were children and adolescents and $>50 \%$ of them came as refugees from countries with devastating civil wars where the exposure may be expected to be high both in the home country and during the difficult journey to Europe. QFT alone is, however, not perfect for diagnosing latent or early active TB because of the late seroconversion in some patients, as shown in two cases in the present sttudy. If resources allow and if compliance for return can be expected to be good, we recommend that both TST and QFT are performed and that patients with positive TST and negative QFT are followed with repeated QFT for at least two years.

The present study can not be used for evaluation of the efficacy of BCG vaccination in neonates or early infancy but it seems obvious that any vaccine efficacy must have waned considerably. This was particularly obvious in Somalians and in unaccompanied minors. Though the children in this study were not systematically questionned about their travelling to Sweden a large number of Somalian and/or unaccompanied minors told spontaneously about several months of being hidden by smugglers in crowded vans, small apartments or tents where the risk of exposure must have been considerable. Many had also spent several months in crowded Libyan, Ethiopian, Turkish and Greek refugee camps. Children from other countries and children who came with adult relatives usually had spent less time and lived under better conditions between leaving the home countries and arrival to Sweden. Another reason for the higher proportion of Quantiferon positivity found in children coming alone or Somalian children could be that these groups had a lower frequency of BCG scars, so it is possible that the vaccination protected them during the first years of life.

\section{Conclusions}

The study showed a significant relation between presence of BCG scar with negative QFT. It did not give a definite answer whether a BCG scar was related to size of TST. The diagnosis of latent TB in health examinations with no known exposure should not be based on a large TST alone but preferably on a combination of TST and QFT. A confirmatory IGRA should be performed in patients with large TST. QFT negative children with large TST should be repeatedly tested by IGRA. The TST diameter was larger 
in QFT positive than in QFT negative children but could not be used to predict the QFT result in the individual patient. Chest $\mathrm{X}$ ray contributes very little to the diagnosis of TB in asymptomatic QFT negative children but should not be omitted because of the possibility of falsely negative QFT or late conversion of QFT in some patients.

\section{Abbreviations}

BCG: Bacillus calmette guérin; CRP: C-Reactive protein test; ESR: Erythrocyte sedimentation rate; IGRA: interferon gamma release assay; ns: Non-significant: NTM: Non-tuberculous mycobacteria; QFT: Quantiferon; TB: Tuberculosis; TST: Tuberculin skin test

\section{Acknowledgements}

We thank the staff at Department of Clinical Bacteriology, Sahlgrenska University Hospital, Gothenburg, Sweden for performing the Quantiferon assays. The study was supported by the Department of Paediatrics, Sahlgrenska University Hospital and Research and the Development Council of the County Göteborg and Södra Bohuslän.

\section{Funding}

Margret Johansson Gudjonsdottir had 10 weeks free time to work with the study financed by the Department of Pediatrics to perform her mandatory scientific work for pediatric speciality. The rest of the work was performed by the authors as part of their clinical work. Compilation of data was performed during the authors' free time.

\section{Availability of data and material}

The ethics committees specifically state that no data which can identify a patient can be publicly available. Even if the database with patients coded is put on the net, it could be possible for someone to identify a patient. The authors can make the data available upon request under code and with omittance of all data which could identify a patient.

\section{Authors' contributions}

MJG participated in designing the study, made several protocols and participated in finalising the manuscript, KK and PW saw a large number of the patients, RSN took most of the blood samples SO was responsible for the study at Angered hospital, DW was responsible for the study at the outpatient clinics in Borås and Alingsås, BT initiated the study, made the final protocol, saw many of the patients, made protocols and finalised the manuscript. All authors read and approved the final manuscript.

\section{Competing interests}

The authors declare that they have no competing interests.

\section{Consent for publication}

All authors have seen and agreed to the submitted version of the paper. All who have been acknowledged as contributors or as providers of personal communications have agreed to their inclusion.

\section{Ethics approval and consent to participate}

The study was approved by the Regional Ethical Review Board of Gothenburg University with specific approval that individual consent was not necessary. All tests performed were part of the clinical routine so no extra blood sampling for the study was made.

\section{Author details \\ 'Department of Paediatrics, Institute of Clinical Sciences, Sahlgrenska Academy, Gothenburg University, Gothenburg, Sweden. ${ }^{2}$ Department of Paediatrics, Angered Hospital, Gothenburg, Sweden. ${ }^{3}$ Department of Child Health, South Älvsborg Hospital, Borås, Sweden. ${ }^{4}$ Department of Pediatrics, Neonatology, Sahlgrenska University Hospital, 41685 Gothenburg, Sweden.}

Received: 25 February 2016 Accepted: 27 September 2016 Published online: 06 October 2016

\section{References}

1. Fjällbrant $H$, Ridell M, Larsson LO. BCG scar and tuberculin reactivity in children and adults. Scand J Infect Dis. 2008;40(5):387-92.
2. Mandalakas AM, Kirchner HL, Zhu X, Starke JR. Interpretation of repeat tuberculin skin testing in international adoptees: conversions or boosting. Pediatr Infect Dis J. 2008;27(10):913-9.

3. Rowland K, Guthmann R, Jamieson B, Malloy D. Clinical inquiries. How should we manage a patient with a positive PPD and prior BCG vaccination? J Fam Pract. 2006;55(8):718-20.

4. Basu Roy R, Sotgiu G, Altet-Gómez N, Tsolia M, Ruga E, Velizarova S, Kampmann B. Identifying predictors of interferon- $\gamma$ release assay results in pediatric latent tuberculosis: a protective role of bacillus Calmette-Guerin?: a pTB-NET collaborative study. Am J Respir Crit Care Med. 2012;186(4):378-84.

5. Kobashi Y, Mouri K, Yagi S, Obase Y, Miyashita N, Okimoto N, Matsushima T, Kageoka T, Oka M. Clinical evaluation for diagnosing active TB disease and transitional change of two commercial blood tests. Scand J Infect Dis. 2008; 40(8):629-34.

6. Sleiman R, Al-Tannir M, Dakdouki G, Ziade F, Assi NA, Rajab M. Interpretation of the tuberculin skin test in bacille Calmette-Guérin vaccinated and nonvaccinated school children. Pediatr Infect Dis J. 2007:26(2):134-8.

7. Dheda K, van Zyl SR, Badri M, Pai M. T-cell interferon-gamma release assays for the rapid immunodiagnosis of tuberculosis: clinical utility in high-burden vs. low-burden settings. Curr Opin Pulm Med. 2009;15(3):188-200.

8. Jacobs S, Warman A, Richardson R, Yacoub W, Lau A, Whittaker D, Cockburn S, Verma G, Boffa J, Tyrrell G, Kunimoto D, Manfreda J, Langlois-Klassen D, Long R. The tuberculin skin test is unreliable in school children BCGvaccinated in infancy and at low risk of tuberculosis infection. Pediatr Infect Dis J. 2011;30(9):754-8.

9. Bianchi L, Galli L, Moriondo M, Veneruso G, Becciolini L, Azzari C, Chiappini E, de Martino $M$. Interferon-gamma release assay improves the diagnosis of tuberculosis in children. Pediatr Infect Dis J. 2009;28(6):510-4.

10. Adetifa IM, Lugos MD, Hammond A, Jeffries D, Donkor S, Adegbola RA, Hill PC. Comparison of two interferon gamma release assays in the diagnosis of Mycobacterium tuberculosis infection and disease in The Gambia. BMC Infect Dis. 2007;7:122

11. Thomas B, Pugalenthi A, Patel H, Woltmann G, Bankart J, Hoskyns W. Concordance between tuberculin skin test and interferon- $\gamma$ assay and interferon- $\gamma$ response to mitogen in pediatric tuberculosis contacts. Pediatr Pulmonol. 2011:46(12):1225-32

12. Pareek M, Bond M, Shorey J, Seneviratne S, Guy M, White P, Lalvani A, Kon OM. Community-based evaluation of immigrant tuberculosis screening using interferon $\gamma$ release assays and tuberculin skin testing: observational study and economic analysis. Thorax. 2013;68(3):230-9.

13. Miramontes R, Hill AN, Yelk Woodruff RS, Lambert LA, Navin TR, Castro KG, LoBue PA. Tuberculosis Infection in the United States: Prevalence Estimates from the National Health and Nutrition Examination Survey, 2011-2012. PLoS One. 2015;10(11)

14. Pareek M, Baussano I, Abubakar I, Dye C, Lalvani A. Evaluation of immigrant tuberculosis screening in industrialized countries. Emerg Infect Dis. 2012; 18(9):1422-1429:e0140881.

15. Alvarez GG, Gushulak B, Abu Rumman K, Altpeter E, Chemtob D, Douglas P, Erkens C, Helbling P, Hamilton I, Jones J, Matteelli A, Paty MC, Posey DL, Sagebiel D, Slump E, Tegnell A, Valín ER, Winje BA, Ellis E. A comparative examination of tuberculosis immigration medical screening programs from selected countries with high immigration and low tuberculosis incidence rates. BMC Infect Dis. 2011;11:3.

16. Santiago EM, Lawson E, Gillenwater K, Kalangi S, Lescano AG, Du Quella G, Cummings K, Cabrera L, Torres C, Gilman RH. A prospective study of Bacillus Calmette-Guérin scar formation and tuberculin skin test reactivity in infants in Lima. Peru Pediatrics. 2003;112(4):e298.

17. Kheir AE, Alhaj AA, Ibrahim SA. The sensitivity of BCG scar as an indicator of previous vaccination among Sudanese infants. Vaccine. 2011;29(46):8189-91.

18. Trollfors B, Stangebye-Nielsen R, Karlsson E, Jönsson B, Dotevall L. Spread of tuberculosis in a high school. Acta Paediatr. 2013;102(3):140-1.

19. Chadha VK, Jagannatha PS, Kumar P. Can BCG-vaccinated children be included in tuberculin surveys to estimate the annual risk of TB infection in India? Int J Tuberc Lung Dis. 2004;8(12):1437-42.

20. Piñeiro R, Mellado MJ, Cilleruelo MJ, García-Ascaso M, Medina-Claros A, GarcíaHortelano M. Tuberculin skin test in bacille Calmette-Guérin-vaccinated children: how should we interpret the results? Eur J Pediatr. 2012;171(11):1625-32.

21. Maeda T, Banno S, Maeda S, Naniwa T, Hayami Y, Watanabe M, Sato S, Ueda R. Comparison of QFT-TB Gold and the tuberculin skin test for detecting previous tuberculosis infection evaluated by chest $C T$ findings in Japanese rheumatoid arthritis patients. J Infect Chemother. 2011;17(6):842-8. 
22. Channabasavaiah R, Murali MV, Suryanarayana HV, Murthy K, Shashidhara AN. Waning of BCG scar and its implications. Indian J Tuberculosis. 1993;40:137-44.

23. Shann F. Different strains of Bacillus Calmette-Guérin vaccine have very different effects on Tuberculosis and on unrelated infections. Clin Infect Dis. 2015;61:960-2

24. Anderson EJ, Webb EL, Mawa PA, Kizza M, Lyadda N, Nampijja M, Elliott AM. The influence of BCG vaccine strain on mycobacteria-specific and nonspecific immune responses in a prospective cohort of infants in Uganda. Vaccine. 2012;30:2083-9.

25. Qian F, Wang W, Qiu Z, Shen Y, He J, Li D, Zhong J, Dai L. Evaluation of a new tuberculosis-related interferon gamma release assay for tuberculosis infection diagnosis in Huzhou, eastern China. Indian J Pathol Microbiol. 2013;56:125-8

26. Khalil KF, Ambreen A, Butt T. Comparison of QuantiFERON-TB Gold Test and Tuberkulin Skin Test in active pulmonary tuberculosis. J Coll Physicians Surg Pak. 2013;23:633-6.

27. Cağlayan V, Ak O, Dabak G, Damadoğlu E, Ketenci B, Ozdemir M, Ozer S, Saygı A. Comparison of tuberculin skin testing and QFT-TB Gold-In Tube test in health care workers. Tuberk Toraks. 2011;59:43-7.

28. Winje BA, Oftung F, Korsvold GE, Mannsåker T, Ly IN, Harstad I, Dyrhol-Riise AM, Heldal E. School based screening for tuberculosis infection in Norway: comparison of positive tuberculin skin test with interferon-gamma release assay. BMC Infect Dis. 2008;8:140.

\section{Submit your next manuscript to BioMed Central} and we will help you at every step:

- We accept pre-submission inquiries

- Our selector tool helps you to find the most relevant journal

- We provide round the clock customer support

- Convenient online submission

- Thorough peer review

- Inclusion in PubMed and all major indexing services

- Maximum visibility for your research

Submit your manuscript at www.biomedcentral.com/submit 